\title{
ECONOMIES OF MANGO CULTIVATION IN A WHOLE-FARM APPROACH FOR SMALLHOLDER FARMERS: A CASE STUDY FROM INDIA
}

\author{
Mehjabeen*, Saravanadurai A
}

Department of economics, Periyar University, Salem, Tamil Nadu, India

Received - November 22, 2020; Revision - June 21, 2021; Accepted - July 29, 2021

Available Online - October 20, 2021

DOI: http://dx.doi.org/10.18006/2021.9(Spl-3-NRMCSSA_2021).S286.S296

\section{KEYWORDS \\ Agricultural economics \\ Horticulture \\ Intercropping \\ Returns on investment \\ Welfare economics}

\begin{abstract}
This case study is about farming systems followed by small mango growers of a Srinivaspur sub-district of Kolar district in Karnataka, India. Over the years, the size of landholdings decreased and suitability has become an issue. The integrated farming system is mostly desired but, the kind of cropping pattern which would bring profitability and sustainability for smallholding farmers under dryland conditions has not been extensively explored. This research analyzes and explains the economies of scale and scope for the smallholder mango growers both in irrigated and rain-fed conditions. The data covers the period from April 2016 to March 2017. Total, 320 smallholder mango growers from Srinivaspur; a sub-district of Kolar in India were randomly interviewed in person, using a structured pre-tested interview schedule. Suitable analytical techniques were used with the data obtained. Further, the results of the study suggested the optimum farming pattern to enhance the income and bring more sustainability to the farmers both in rain-fed and irrigated conditions.
\end{abstract}

* Corresponding author

E-mail: mehjabeen.nish@gmail.com (Mehjabeen)

Peer review under responsibility of Journal of Experimental Biology and Agricultural Sciences.

Production and Hosting by Horizon Publisher India [HPI] (http://www.horizonpublisherindia.in/).

All rights reserved.
All the articles published by Journal of Experimental Biology and Agricultural Sciences are licensed under a Creative Commons Attribution-NonCommercial 4.0 International License Based on a work at www.jebas.org.






\section{Introduction}

In India, about $84 \%$ of small and marginal farmers carry 115 million agricultural operations as over the years the size of landholdings became small (Gill, 2009; Singh et al., 2011). India has its cultivation only on $29 \%$ of such arable land which has poor resources and adverse climatic conditions. Smallholding farmers are less benefited by the advances in agriculture and the wellestablished farmers are major takers of such benefits. Cropping has not been satisfactory for running the livelihood of the average farmers' family. The concerning factor is the livelihoods of the small and marginal household families. Many farming systems came into the picture aiming at using the waste generated from the farming and animal raring activities resulting in more depletion of resources (Singh et al., 2011).

Agriculture is an industry that demands a lot of water consumption. The integrated farming system is a judicious mix of various farming systems. It provides scope for increasing water efficiency and the socio-economic condition of the farmers. It was introduced in Asia which slowly gained popularity in the rest of the world (Behera, 2012).

The integrated farming system maximizes the productivity of a farm by optimum utilization of resources and better residual management. It has been proved to be one of the sustainable agricultural practices for dry-land farmers in comparison to the traditional cropping system which is highly uncertain in terms of productivity, employment, and income (Radhamani, 2003). Research scholars from various agricultural universities at the state level, affiliated with the Indian Council of Agricultural Research (ICAR) has studied individually many agricultural operations such as goat keeping, dairy, duck keeping, livestock, apiculture, poultry, sericulture, horticulture, goat keeping, mushroom cultivation, piggery, etc. to enhance the productivity of a farm but as it lacks sustainability for smallholder farmers as the integrated approach of all these farming systems remained missing in those researches as farmers do not follow the integrated approach of the farming system. The integrated farming system is a need of the hour. It should be interrelated where residual or the byproduct from one enterprise could be used for the other enterprise with an intense complementary effect which would form a cycle and the input use efficiency keeps escalating and improves the productivity by about $50 \%$. Hence, the research focused on sustainability, improving productivity and profitability by providing the solution for waste management, regular income, generation of employment, and meeting the livelihood requirements of the farmers (Gill, 2009). The literature review has not given us any related study on the optimum cropping pattern for dry land horticulture small farmers under irrigated and rain-fed conditions.
Vegetable demands are highly price-elastic for low-income and middle-income consumers (Motkuri, 2020). Growing and selling vegetables could be profitable based on market demand. For sustainability, the cropping pattern should be packed with the cultivation of other crops too. Zero budget natural farming (ZBNF) is a natural way of organic farming where Indian domestic cow urine and dung are used for cultivation without any external inputs and it also has a process of cultivation that has to be followed. It has proven to enhance the yield of crops (Khadse \& Peter, 2019).

India is the largest producer of mango and contributes about $50 \%$ of the world's mango production. Mango contributes about $40 \%$ of the total fruit production of the country. Mango is a perennial fruit crop that has showed the highest return (Mehjabeen \& Saravanadurai, 2020). In India, Uttar Pradesh is the largest producer of mango, followed by Andhra Pradesh and Karnataka (Indian Horticulture Database, 2011; The Director of Statistics, 2015). Srinivaspur of Kolar district in Karnataka was categorized as dry land which showed the highest trend in mango production among other sub-districts in Karnataka. It is also termed as the Land of World Famous Mangoes. About $50 \%$ of the total production in the district comes from Srinivaspur and about $90 \%$ of the farmers are in the taluk are mango growers (Mehjabeen \& Saravanadurai, 2020; National Mango Database (2020).

There are crops like legumes that compliment the environment and enrich the soil. They are complementary crops to the other crops grown along. It is also a good source of farmers' income. Legumes fix 200 to $300 \mathrm{~kg}$ of nitrogen per hectare. Legumes are estimated to fix $50-70 \%$ of biological nitrogen in the world (Devi, 2016). It means it fixes 80 to $120 \mathrm{~kg}$ of nitrogen per acre that works out to be INR 786.67 .

Eucalyptus commonly known as Nilgiri does not allow other plants to grow near it. It consumes 20-40 liters of water a day and it also drastically affects the groundwater level (Shetty, 2019), therefore, it can not be considered for dryland farming and also for intercropping. Tamarind trees stand about $24 \mathrm{~m}$ tall and cover about $7 \mathrm{~m}$ of the surface which makes them cover a large area. They also yield the most acidic fruit ever found (Mathew \& Rao, 2012). Therefore, it might not be recommended for intercropping. This case study is about farming systems followed by small mango growers of a Srinivaspur sub-district in India

\section{Materials and Methods}

The study is based on primary data which was collected at Srinivaspur, a sub-district of Kolar district in Karnataka in peninsula India in April 2017. It includes the period from March 2016 to April 2017. A total of 320 smallholding mango cultivating 
farmers were randomly interviewed. The sample size was decided based on the population of smallholder farmers in the sub-district i.e. 7452 (Shivakumar \& Chandra, 2016) with a confidence level of $95 \%$ and confidence interval of 5.36. A structured interview schedule has been used to collect the data. There are 5 hobblies in the sub-district. Among the selected areas, 3 villages were visited in each hobblie and interviewed 20 smallholding mango cultivating farmers from each village randomly. 20 farmers who have visited the horticulture department located in the Srinivaspur town during the time of data collection were also interviewed. We followed two parameters while considering the sample to contribute towards our data (1) small landholders i.e. who have land holdings from 2.5 to 5 acres and; (2) farmers whose $50 \%$ of the total income is from mango cultivation. During the survey, the Srinivaspur area was surveyed because it is specialized in mango cultivation and is a dry land area. This study attempted to know the best combination of farming or the best crop mix which would bring sustainability, productivity, and profitability for the smallholder mango farmers in both irrigated and rain-fed conditions. During the process, this study also explored how it could be environmentally friendly. The study also calculated the total cost of the input, gross returns, net returns, and returns on investments of an individual enterprise of each respondent. In this indirect net returns was also considered for those respondents who have cropped horse gram for manure purposes. The formulae used directly in this paper are

- Total Cost $=$ Total Fixed Cost + Total Variable Cost.

- Gross Returns $=$ sum of all receipts from the main and byproduct

- $\quad$ Net Returns*= Gross Returns - Total cost

- $\quad$ Returns on Investment $=$ Net Returns/Total cost $($ Chen, 2020; Beattie, 2020)

While indirect formulae used in the current study are

- Depreciation per year on farm implements/ machineries/ Livestock shed $=($ present vale- salvage value $) /$ Expected life (Zarzycki, 2020).

- $\quad$ Appreciation of livestock per year $=($ Cull value - Purchase value)/ average life span. (The apportionment cost of a Hybrid cow is 50000 INR. The government provides loans and there is no interest in fixed capital. However, the farmers are required to repay the premium @ 2000 INR/ Month which is $24000 \mathrm{INR} /$ year)

- Establishment cost and maintenance cost for a perennial crop such as mango, papaya, coconut, and tamarind have amortized on the gestation period of the tree.
- $\quad *$ Electricity cost for irrigation per year $=($ Motor power in $\mathrm{HP} \times 0.746) \times(3$ hours per irrigation $) \times(7 \mathrm{INR}) \times$ Number of irrigations in a year (*When Horse Power (HP) is given, if multiplied with 0.746 we will get electricity consumption of motor per hour in Kilowatts - Smathers, 2017).

Further, we categorized them based on the combination of the enterprise of their farming pattern. All the obtained data were analyzed and tables were constructed using Microsoft excel 2016. Further, arithmetic means, percentage method, and average priority sorting have been used for showing results.

\section{Results and discussion}

From table 1, it is evident that $86.6 \%$ of respondents are educated which shows the literacy rate is high in the taluk. Among these, $69.4 \%$ of the respondents fall in the age group of 30 and 1-60 which, is considered to be a productive age. Further, $75.6 \%$ of the respondents are Hindu and $24.4 \%$ are Muslim. The main occupation of the respondents is agriculture, and $50.6 \%$ of the respondents have no subsidiary occupation. $\mathrm{TV}$ is the main source of information and farm education among respondents followed by TV \& Radio. The respondents do not prefer visiting KVKs for the information and $55.9 \%$ of the respondents have around 3 acres of land. Out of 129 irrigated respondents, 124 have borewell and the other 5 borrows water from those who have borewell which makes borewell as the sole source of irrigation. Only $9.4 \%$ of the respondents have opted for farm pond or krishi vonda which is very less compared to the drip set adopters of $38.8 \%$. The farm pond is not more too old scheme and farmers failed to understand its benefits, which might be the reason for its very low adoption rate.

In the case of farm machinery, $85.6 \%$ of the respondents hold farm implements and machinery whereas only $4.1 \%$ have a bullock cart which shows adoption and implementation of modern machinery is high. $77.2 \%$ and $1.6 \%$ of respondents hold 2 wheelers and 4 wheelers respectively, for personal use whereas, $0.9 \%$ of respondents hold 3 wheelers which is the source of their subsidiary income. In living conditions, $97.5 \%$ of respondents own their houses and $96.6 \%$ have flat-stone rooftops which, shows their living standards is good. From table 2 we could see 77 and 194 of respondents have sheep and goat and cow and buffaloes, respectively, out of which 186 have cow/sheep shed which is lesser comparatively. $27.2 \%$ of the respondents are beneficiaries of government policies. Only $4.4 \%$ of respondents insured their mangos and $2.8 \%$ insured their tomatoes and ragi for the season which shows insurance is not much popular in the taluk. 
Table 1 Descriptive analysis

\begin{tabular}{|c|c|c|c|c|}
\hline S No & Particulars & Valid & Frequency & Percent \\
\hline \multirow{2}{*}{1} & \multirow{2}{*}{ Irrigated/ Rain-fed } & Irrigated & 129 & 40.31 \\
\hline & & Rainfed & 191 & 59.69 \\
\hline \multirow{2}{*}{2} & \multirow{2}{*}{ Gender of respondents } & Male & 304 & 95 \\
\hline & & Female & 16 & 5 \\
\hline \multirow{3}{*}{3} & \multirow{3}{*}{ Age } & 30 and below & 10 & 3.1 \\
\hline & & $30.1-60$ & 222 & 69.4 \\
\hline & & 60.1 and above & 88 & 27.5 \\
\hline \multirow{8}{*}{4} & \multirow{8}{*}{ Educational qualification } & Illiterate & 43 & 13.4 \\
\hline & & Primary & 83 & 25.9 \\
\hline & & Secondary & 66 & 20.6 \\
\hline & & Diploma & 2 & 0.6 \\
\hline & & SSLC & 81 & 25.3 \\
\hline & & PUC & 24 & 7.5 \\
\hline & & Graduation & 19 & 5.9 \\
\hline & & $\mathrm{PhD}$ & 2 & 0.6 \\
\hline \multirow{2}{*}{5} & \multirow{2}{*}{ Religion } & Hindu & 242 & 75.6 \\
\hline & & Muslim & 78 & 24.4 \\
\hline \multirow{3}{*}{6} & \multirow{3}{*}{ Category } & General & 134 & 41.9 \\
\hline & & $\mathrm{OBC}$ & 138 & 43.1 \\
\hline & & $\mathrm{SC} / \mathrm{ST}$ & 48 & 14 \\
\hline \multirow{2}{*}{7} & \multirow{2}{*}{ Family type } & Nuclear & 277 & 86.6 \\
\hline & & Joint & 43 & 13.4 \\
\hline \multirow{2}{*}{8} & \multirow{2}{*}{ Main Occupation } & Agriculture & 316 & 98.8 \\
\hline & & Services & 4 & 1.3 \\
\hline \multirow{5}{*}{9} & \multirow{5}{*}{ Subsidiary occupation } & Non & 162 & 50.6 \\
\hline & & Labourer & 84 & 26.3 \\
\hline & & Other Services & 27 & 8.4 \\
\hline & & Agriculture & 3 & 0.9 \\
\hline & & Business & 44 & 13.8 \\
\hline \multirow{2}{*}{10} & \multirow{2}{*}{ Health Care facilities } & Yes & 320 & 100 \\
\hline & & No & 0 & 0 \\
\hline \multirow{2}{*}{11} & \multirow{2}{*}{ Veterinary Hospital } & Yes & 320 & 100 \\
\hline & & No & 0 & 0 \\
\hline \multirow{4}{*}{12} & \multirow{4}{*}{ Source of farmers' education } & TV & 293 & 91.6 \\
\hline & & TV \& KVK & 1 & 0.3 \\
\hline & & TV \& Radio & 25 & 7.8 \\
\hline & & TV, Radio \& KVK & 1 & 0.3 \\
\hline
\end{tabular}




\begin{tabular}{|c|c|c|c|c|}
\hline S No & Particulars & Valid & Frequency & Percent \\
\hline \multirow{3}{*}{13} & \multirow{3}{*}{ Land size } & Less than or equal to 3 acre & 179 & 55.9 \\
\hline & & 3.1 to 4 acres & 65 & 20.3 \\
\hline & & 4.1-5 acres & 76 & 23.8 \\
\hline \multirow{2}{*}{14} & \multirow{2}{*}{ Bore Well } & Yes & 124 & 38.8 \\
\hline & & No & 196 & 61.3 \\
\hline \multirow{2}{*}{15} & \multirow{2}{*}{ Drip set } & Yes & 120 & 37.5 \\
\hline & & No & 200 & 62.5 \\
\hline \multirow{2}{*}{16} & \multirow{2}{*}{ Krishi Vonda or Farm Pond } & Yes & 30 & 9.4 \\
\hline & & No & 290 & 90.6 \\
\hline \multirow{2}{*}{17} & \multirow{2}{*}{ Farm Implements and Machinery } & Yes & 274 & 85.6 \\
\hline & & No & 46 & 14.4 \\
\hline \multirow{2}{*}{18} & \multirow{2}{*}{ Bullock Cart } & No & 307 & 95.9 \\
\hline & & Yes & 13 & 4.1 \\
\hline \multirow{2}{*}{19} & \multirow{2}{*}{2 Wheeler } & Yes & 247 & 77.2 \\
\hline & & No & 73 & 22.8 \\
\hline \multirow{2}{*}{20} & \multirow{2}{*}{3 Wheeler } & Yes & 3 & 0.9 \\
\hline & & No & 317 & 99.1 \\
\hline \multirow{2}{*}{21} & \multirow{2}{*}{4 Wheeler } & Yes & 5 & 1.6 \\
\hline & & No & 315 & 98.4 \\
\hline \multirow{2}{*}{22} & \multirow{2}{*}{ House (Owned) } & Yes & 312 & 97.5 \\
\hline & & No & 8 & 2.5 \\
\hline \multirow{3}{*}{23} & \multirow{3}{*}{ Type of house } & Temporary roof & 3 & 0.9 \\
\hline & & Flat stone roof & 309 & 96.6 \\
\hline & & $\mathrm{RCC}$ & 8 & 2.5 \\
\hline \multirow{2}{*}{24} & \multirow{2}{*}{ Cow/Sheep Shed } & No & 134 & 41.9 \\
\hline & & Yes & 186 & 58.1 \\
\hline \multirow{2}{*}{25} & \multirow{2}{*}{ Government policies/Schemes benefits } & No & 233 & 72.8 \\
\hline & & Yes & 87 & 27.2 \\
\hline \multirow{2}{*}{26} & \multirow{2}{*}{ Crop Insurance for Mango } & No & 306 & 95.6 \\
\hline & & Yes & 14 & 4.4 \\
\hline \multirow{3}{*}{27} & & No Crops & 311 & 97.2 \\
\hline & Crop insurance(other Crops) & Tomato & 3 & 0.9 \\
\hline & & Ragi & 6 & 1.9 \\
\hline & & No & 198 & 61.9 \\
\hline 20 & 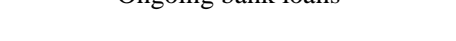 & Yes & 122 & 38.1 \\
\hline
\end{tabular}

Source: Survey 2017 
Table 2 Yield of crops in kg per acre and market-rate in INR per kg

\begin{tabular}{|c|c|c|c|c|c|c|c|c|c|c|c|}
\hline \multirow{2}{*}{$\begin{array}{c}\text { S } \\
\text { No }\end{array}$} & \multirow{2}{*}{$\begin{array}{l}\text { Crop } \\
\text { category }\end{array}$} & \multirow[t]{2}{*}{ Crop Name } & \multirow{2}{*}{$\begin{array}{c}\text { No of } \\
\text { Respondents }\end{array}$} & \multicolumn{2}{|c|}{$\begin{array}{l}\text { Market-rate } \\
\text { INR/kg }\end{array}$} & \multirow{2}{*}{$\begin{array}{c}\text { (IRR) No. } \\
\text { of } \\
\text { Respondents }\end{array}$} & \multicolumn{2}{|c|}{$\begin{array}{l}\text { Yield kg/Acre- } \\
\text { irrigated }\end{array}$} & \multirow{2}{*}{$\begin{array}{l}\text { (RF) No. of } \\
\text { Respondents }\end{array}$} & \multicolumn{2}{|c|}{$\begin{array}{l}\text { Yield Kg/Acre- } \\
\text { Rain fed }\end{array}$} \\
\hline & & & & Main & By & & Main & By & & Main & By \\
\hline 1 & $\begin{array}{l}\text { Mango } \\
\text { (Fruit) }\end{array}$ & Mango & $320(100 \%)$ & 17 & - & $\begin{array}{c}129 \\
(40.31 \%) \\
\end{array}$ & 6541 & - & $\begin{array}{c}191 \\
(59.69 \%) \\
\end{array}$ & 4980 & - \\
\hline 2 & \multicolumn{2}{|c|}{ Other fruits } & \multicolumn{3}{|c|}{$2(0.63 \%)$} & \multicolumn{3}{|c|}{$2(0.63 \%)$} & \multicolumn{3}{|c|}{0} \\
\hline 2.1 & $\begin{array}{l}\text { Other } \\
\text { Fruits } \\
\end{array}$ & Papaya & 1 & 10 & - & 1 & 25000 & - & 0 & - & - \\
\hline 2.2 & $\begin{array}{l}\text { Other } \\
\text { Fruits }\end{array}$ & Watermelon & 1 & 8 & - & 1 & 32000 & - & 0 & - & - \\
\hline 3 & \multicolumn{2}{|c|}{ Cereals } & \multicolumn{3}{|c|}{$195(60.94 \%)$} & \multicolumn{3}{|c|}{$79(24.69 \%)$} & \multicolumn{3}{|c|}{$116(36.25 \%)$} \\
\hline 3.1 & Cereals & Ragi & 188 & 50 & 1 & 73 & 1335.74 & 2500 & 115 & 975 & 2500 \\
\hline 3.2 & Cereals & Jowar $^{*}$ & 36 & 25 & 1 & 19 & 2666.67 & 666.67 & 17 & 4000 & 1000 \\
\hline 3.3 & Cereals & Paddy & 7 & 18 & 1 & 7 & 620 & 500 & 0 & - & - \\
\hline 3.4 & Cereals & $\begin{array}{c}\text { Little } \\
\text { Millets }\end{array}$ & 2 & 30 & 1 & 0 & - & - & 2 & 500 & 1000 \\
\hline 3.5 & Cereals & $\begin{array}{l}\text { Hybrid } \\
\text { maize }^{*}\end{array}$ & 1 & 15 & 1 & 0 & 3200 & 8000 & 1 & 3700 & 12000 \\
\hline 3.6 & Cereals & Corn $^{*}$ & 1 & 12 & 1 & 0 & - & - & 1 & 4000 & 1000 \\
\hline 4 & \multicolumn{2}{|c|}{ Pulses } & \multicolumn{3}{|c|}{$182(56.88 \%)$} & \multicolumn{3}{|c|}{$63(19.69 \%)$} & \multicolumn{3}{|c|}{$119(37.19 \%)$} \\
\hline 4.1 & Pulses & Field beans & 158 & 30 & 30 & 60 & 741.26 & 174.16 & 98 & 650.57 & 162.41 \\
\hline 4.2 & Pulses & Horse gram & 66 & 30 & - & 14 & 168.80 & - & 52 & 286.21 & - \\
\hline 4.3 & Pulses & Red gram & 26 & 80 & - & 14 & 1590.91 & - & 12 & 2173.33 & - \\
\hline 5 & Planta & on Crop & $18(5$ & $63 \%)$ & & & $1.88 \%)$ & & & $3.75 \%)$ & \\
\hline 5.1 & $\begin{array}{c}\text { Plantation } \\
\text { Crop }\end{array}$ & Coconut $^{* *}$ & 1 & 15 & - & 0 & 80 & - & 1 & - & - \\
\hline 5.2 & $\begin{array}{c}\text { Plantation } \\
\text { Crop } \\
\end{array}$ & Tamarind $^{* *}$ & 15 & 40 & - & 4 & - & - & 11 & 40 & - \\
\hline 5.3 & $\begin{array}{c}\text { Plantation } \\
\text { Crop }\end{array}$ & Eucalyptus & 2 & 5 & - & 1 & 16200 & - & 1 & 16000 & - \\
\hline 5.4 & $\begin{array}{c}\text { Plantation } \\
\text { Crop } \\
\end{array}$ & Sugarcane & 1 & 10 & - & 1 & 24000 & - & 0 & - & - \\
\hline 6 & & wer & $6(1$ & $38 \%)$ & & & $1.88 \%)$ & & & 0 & \\
\hline 6.1 & Flower & Marigold & 6 & 60 & - & 6 & 3309.091 & - & 0 & - & - \\
\hline 7 & Veg & tables & $93(2$ & $.06 \%)$ & & & $29.06 \%)$ & & & 0 & \\
\hline 7.1 & Vegetables & tomato & 89 & 10 & - & 89 & 20993.23 & - & 0 & - & - \\
\hline 7.2 & Vegetables & chili & 15 & 30 & - & 15 & 808.33 & & 0 & & \\
\hline 7.3 & Vegetables & coriander & 14 & 5.5 & 45 & 14 & 3928 & 164 & 0 & - & - \\
\hline 7.4 & Vegetables & cauliflower & 12 & 10 & - & 12 & 8412.70 & - & 0 & - & - \\
\hline 7.5 & Vegetables & potato & 11 & 20 & - & 11 & 6346.15 & - & 0 & - & - \\
\hline 7.6 & Vegetables & Brinjal & 7 & 13.09 & - & 7 & 8380.95 & - & 0 & - & - \\
\hline 7.7 & Vegetables & $\begin{array}{l}\text { Ridge } \\
\text { gourd }\end{array}$ & 6 & 20 & - & 6 & 3000 & - & 0 & - & - \\
\hline 7.8 & Vegetables & carrot & 4 & 30 & - & 4 & 6250 & - & 0 & - & - \\
\hline 7.9 & Vegetables & cabbage & 4 & 10 & - & 4 & 9600 & - & 0 & - & - \\
\hline 7.10 & Vegetables & bitter gourd & 3 & 25 & - & 3 & 3733.33 & - & 0 & - & - \\
\hline 7.11 & Vegetables & Onion & 2 & 4 & - & 2 & 4000 & - & 0 & - & - \\
\hline 7.12 & Vegetables & Beetroot & 2 & 10 & - & 2 & 10000 & - & 0 & - & - \\
\hline 7.13 & Vegetables & cucumber & 2 & 10 & - & 2 & 5600 & - & 0 & - & - \\
\hline 7.14 & Vegetables & pumpkin & 2 & 8 & - & 2 & 12000 & - & 0 & - & - \\
\hline 7.15 & Vegetables & snake gourd & 1 & 40 & - & 1 & 10000 & - & 0 & - & - \\
\hline 7.16 & Vegetables & bottle gourd & 1 & 12 & - & 1 & 12000 & - & 0 & - & - \\
\hline 7.17 & Vegetables & Mint & 1 & 3.13 & - & 1 & 8000 & - & 0 & - & - \\
\hline 7.18 & Vegetables & Capsicum & 1 & 40 & - & 1 & 7500 & - & 0 & - & - \\
\hline 7.19 & Vegetables & Garlic & 1 & 30 & - & 1 & 3000 & - & 0 & - & - \\
\hline 8 & Mulberry & Mulberry & $11(3.44 \%)$ & 14 & - & $11(3.44 \%)$ & 13851.85 & - & 0 & - & - \\
\hline
\end{tabular}

Survey 2017; Note: *States crops grown for green fodder; **States yield per tree 
Cereals and pulses are largely grown by rain-fed respondents compared to irrigated respondents (Table 2). Respondents for plantation crops are also more in rain-fed than in irrigated conditions. However, other fruits, vegetables, flowers, and mulberry are only grown by respondents with the irrigated condition. Ragi is largely grown in cereals, followed by jowar, paddy, little millet, hybrid maize, and corn. Jowar, hybrid maize, and corn are grown to serve the requirement of green fodder for the cattle whereas, straw of ragi, paddy, and little millets are used as dry fodder. Field beans are widely grown in pulses followed by horse-gram and red-gram. Cereals and pulses are mainly grown for serving the family's food needs of respondents. In plantation crops, tamarind is widely grown. It is also used as an ingredient for cooking. Respondents sell it in the market after keeping it for home use. In flowers, marigold is grown especially during festival season to get good returns. In vegetables, tomatoes are widely grown for commercial purposes followed by chilly, coriander, potatoes, and so on. Respondents who rear silkworms grow mulberry whereas, few of them grow it for commercial purposes.

Respondents who tame cows and sheep and goats are more in rainfed conditions than respondents in irrigated conditions (Table $3)$. Respondents in the rain-fed condition that rear sheep and goats and hybrid cows are $60.42 \%$ and $57.78 \%$ more than respondents with irrigated condition respectively. It shows sheep and goat rearing is popular among rain-fed respondents. Domestic cows are tamed by very few respondents whereas; hybrid cows (Holstein
Friesian or the HF) are popular among them. Buffaloes and bullocks are tamed more by respondents in irrigated conditions than rain-fed one. Silkworms are reared by the only mulberry cultivating respondents. $55.63 \%$ and $24.06 \%$ of respondents depend on cows and buffaloes and sheep and goats for meeting their regular expenses which, makes livestock as their lifeline.

Crops such as snake-gourd followed by capsicum, watermelon, papaya, sugarcane, tomato, marigold, mulberry, carrot, bottle gourd, red grams, and potato have more gross return than mango as they are grown commercially (Table 4). However, cereals and pulses do not have high gross returns. From table 5, it is revealed that mango has the highest Returns on Investment of respondents as we have considered those farmers whose $50 \%$ of the total earnings come from mango. It also has the lowest variable cost than other combinations of cropping patterns. However, the net returns are more for those combinations which have livestock and plantation crops for respondents under both rain-fed and irrigated conditions. These results are in agreement with the data available at APEDA (2020). The literature review has also shown us that better residual management is not possible without livestock rearing. Respondents under irrigated conditions who rear silkworms also grow mulberry which is much feasible and has high net returns. Cereals and pulses are grown for serving the family food requirement and as well as dry fodder needs of the cattle so it is grown in both rain-fed and irrigated condition. A cropping pattern with other fruits showed the highest net returns (Table 6).

Table 3 Livestock's yield in kg per acre and market rate in INR per kg

\begin{tabular}{|c|c|c|c|c|c|c|c|c|c|c|}
\hline \multirow{2}{*}{$\begin{array}{c}\text { S } \\
\text { No }\end{array}$} & \multirow{2}{*}{ Category } & \multirow{2}{*}{ Variety } & \multirow{2}{*}{$\begin{array}{l}\text { No of IRR } \\
\text { respondents }\end{array}$} & \multirow{2}{*}{ RF } & \multicolumn{3}{|c|}{ Market value in INR } & \multicolumn{3}{|c|}{ The quantity obtained in $\mathrm{Kg}$} \\
\hline & & & & & Main 1 & Main 2 & By & Main 1 & Main 2 & By \\
\hline 1 & Cow\& Buffal & $(178)(55.63 \%)$ & $80(25 \%)$ & $98(30.63 \%)$ & & & & & & \\
\hline 1.1 & Cow\& Buffalo & Domestic cow & 7 & 13 & 29 & 150 & 1.6 & 1626 & 95 & 3000 \\
\hline 1.2 & Cow\& Buffalo & Domestic Heifer & 2 & 3 & - & 150 & 1.6 & - & 85 & 3000 \\
\hline 1.3 & Cow\& Buffalo & Domestic Calf & 10 & 11 & - & 150 & 1.6 & - & 40 & 1500 \\
\hline 1.4 & Cow\& Buffalo & Hybrid cow & 26 & 45 & 29 & 150 & 1.6 & 8763.94 & 130 & 4000 \\
\hline 1.5 & Cow\& Buffalo & Hybrid Heifer & 9 & 9 & - & 150 & 1.6 & - & 100 & 3000 \\
\hline 1.6 & Cow\& Buffalo & Hybrid calf & 53 & 54 & - & 150 & 1.6 & - & 50 & 2000 \\
\hline 1.7 & Cow\& Buffalo & Buffalo & 22 & 18 & 29 & - & 1.6 & 1364.29 & 200 & 4000 \\
\hline 1.8 & Cow\& Buffalo & Buffalo heifer & 3 & 3 & - & - & 1.6 & - & 150 & 3000 \\
\hline 1.9 & Cow\& Buffalo & Buffalo calf & 18 & 11 & - & - & 1.6 & - & 75 & 2000 \\
\hline 1.10 & Cow\& Buffalo & Bullock & 8 & 3 & - & 150 & 1.6 & - & 100 & 4000 \\
\hline 1.11 & $\begin{array}{l}\text { Sheep\& Goat } \\
(77)(24.06 \%)\end{array}$ & Sheep\& Goat & $29(9.06 \%)$ & $48(15 \%)$ & - & 430 & 3 & - & 23 & 2000 \\
\hline 1.12 & $\begin{array}{c}\text { Silkworm } \\
\text { raring (7) } \\
(2.19 \%)\end{array}$ & (100DFL) & $7(2.19 \%)$ & 0 & 518.4 & - & 108 & - & - & - \\
\hline
\end{tabular}

Survey 2017

Journal of Experimental Biology and Agricultural Sciences

http://www.jebas.org 
Table 4 Value in INR for per acre yield

\begin{tabular}{|c|c|c|c|c|}
\hline S No & Crops & No of Respondents & Value (IRR) & Value (RF) \\
\hline 1 & Snake gourd & 1 & 400000 & 0 \\
\hline 2 & Capsicum & 1 & 300000 & 0 \\
\hline 3 & Watermelon & 1 & 256000 & 0 \\
\hline 4 & Papaya & 1 & 250000 & 0 \\
\hline 5 & Sugarcane & 1 & 240000 & 0 \\
\hline 6 & tomato & 89 & 209932.3 & 0 \\
\hline 7 & Marigold & 6 & 198545.5 & 0 \\
\hline 8 & Mulberry & 11 & 193925.9 & 0 \\
\hline 9 & carrot & 4 & 187500 & 0 \\
\hline 10 & bottle gourd & 1 & 144000 & 0 \\
\hline 11 & Red gram & 26 & 127272.8 & 173866.4 \\
\hline 12 & potato & 11 & 126923 & 0 \\
\hline 13 & Mango & 320 & 111197 & 84660 \\
\hline 14 & Brinjal & 7 & 109706.6 & 0 \\
\hline 15 & Beetroot & 2 & 100000 & 0 \\
\hline 16 & cabbage & 4 & 96000 & 0 \\
\hline 17 & pumpkin & 2 & 96000 & 0 \\
\hline 18 & bitter gourd & 3 & 93333.25 & 0 \\
\hline 19 & Garlic & 1 & 90000 & 0 \\
\hline 20 & cauliflower & 12 & 84127 & 0 \\
\hline 21 & Eucalyptus & 2 & 81000 & 80000 \\
\hline 22 & Ragi & 188 & 69287 & 51250 \\
\hline 23 & Jowar* & 36 & 67333.42 & 101000 \\
\hline 24 & Ridge gourd & 6 & 60000 & 0 \\
\hline 25 & Hybrid maize* & 1 & 56000 & 67500 \\
\hline 26 & cucumber & 2 & 56000 & 0 \\
\hline 27 & coriander & 14 & 28984 & 0 \\
\hline 28 & Field beans & 158 & 27462.6 & 24389.4 \\
\hline 29 & Mint & 1 & 25040 & 0 \\
\hline 30 & chili & 15 & 24249.9 & 0 \\
\hline 31 & Onion & 2 & 16000 & 0 \\
\hline 32 & Paddy & 7 & 11660 & 0 \\
\hline 33 & Horse gram & 66 & 5064 & 8586.3 \\
\hline 34 & Coconut** & 1 & 1200 & 0 \\
\hline 35 & Little Millets & 2 & 0 & 16000 \\
\hline 36 & Corn* & 1 & 0 & 49000 \\
\hline 37 & Tamarind ${ }^{* *}$ & 15 & 0 & 1600 \\
\hline
\end{tabular}

Survey 2017; Note: *States crops grown for green fodder; **States yield per tree. 
Table 5 Farming pattern under irrigated condition

\begin{tabular}{|c|c|c|c|c|c|c|c|c|}
\hline $\begin{array}{l}\text { S } \\
\text { No. }\end{array}$ & Farming Pattern & $\begin{array}{c}\text { No. of } \\
\text { Respondents }\end{array}$ & $\mathrm{VC}$ & FC & $\mathrm{TC}^{*}$ & $\mathrm{GR}^{*}$ & NR* & $\begin{array}{c}\text { RO } \\
\text { I }\end{array}$ \\
\hline 1 & $\mathrm{M}+\mathrm{OF}$ & 1 & 96452.4 & 15793.19 & 112,246 & 547,700 & 435,454 & 4 \\
\hline 2 & $\mathrm{M}+\mathrm{PC}+\mathrm{C}+\mathrm{P}+\mathrm{C} \& \mathrm{~B}+\mathrm{S} \& \mathrm{G}$ & 1 & 132357 & 14908 & 147,265 & 426,432 & 279,166 & 2 \\
\hline 3 & $\mathrm{M}+\mathrm{PC}$ & 1 & 36758.6 & 14534.8 & 51,293 & 274,050 & 222,757 & 4 \\
\hline 4 & $\mathrm{M}+\mathrm{V}+\mathrm{C}+\mathrm{P}+\mathrm{C} \& \mathrm{~B}$ & 11 & 143156.56 & 11747.38 & 154,904 & 379,199 & 224,358 & 1 \\
\hline 5 & $\mathrm{M}+\mathrm{C}+\mathrm{P}+\mathrm{Mul}+\mathrm{C} \& \mathrm{~B}+\mathrm{SR}$ & 2 & 114930.32 & 13662.35 & 128,593 & 380544.8 & 251952.2 & 2 \\
\hline 6 & $\mathrm{M}+\mathrm{V}+\mathrm{Mul}+\mathrm{C} \& \mathrm{~B}+\mathrm{SR}$ & 1 & 157316.8 & 21805.38 & 179,122 & 416,039 & 250,971 & 1 \\
\hline 7 & $\mathrm{M}+\mathrm{C}+\mathrm{P}+\mathrm{Mul}+\mathrm{C} \& \mathrm{~B}$ & 2 & 86216.21 & 6931.59 & 93,148 & 285,348 & 192,321 & 2 \\
\hline 8 & $\mathrm{M}+\mathrm{V}+\mathrm{C}+\mathrm{P}$ & 7 & 75634.89 & 9933.04 & 85,568 & 247,581 & 162,090 & 2 \\
\hline 9 & M & 3 & 10436.61 & 15043.21 & 25,480 & 175,997 & 150,517 & 6 \\
\hline 10 & $\mathrm{M}+\mathrm{V}+\mathrm{C}+\mathrm{P}+\mathrm{S} \& \mathrm{G}$ & 1 & 48602.88 & 2240.74 & 50,844 & 212,633 & 161,790 & 3 \\
\hline 11 & $\mathrm{M}+\mathrm{V}+\mathrm{F}+\mathrm{PC}+\mathrm{C}+\mathrm{P}+\mathrm{C} \& \mathrm{~B}+\mathrm{S} \& \mathrm{G}$ & 1 & 212,908 & 16,920 & 229,828 & 536,353 & 306,525 & 1 \\
\hline 12 & $\mathrm{M}+\mathrm{V}+\mathrm{PC}+\mathrm{C}+\mathrm{P}+\mathrm{C} \& \mathrm{~B}$ & 2 & 128,608 & 15,707 & 144,315 & 354,311 & 210,127 & 1 \\
\hline 13 & $\mathrm{M}+\mathrm{OF}+\mathrm{V}+\mathrm{C} \& \mathrm{~B}$ & 1 & 133,790 & 8,221 & 142,011 & 351,668 & 209,657 & 1 \\
\hline 14 & $\mathrm{M}+\mathrm{V}+\mathrm{C} \& \mathrm{~B}+\mathrm{S} \& \mathrm{G}$ & 6 & 107,227 & 12,424 & 119,651 & 296,079 & 176,428 & 1 \\
\hline 15 & $\mathrm{M}+\mathrm{C}+\mathrm{C} \& \mathrm{~B}$ & 4 & 69,278 & 8,762 & 78,040 & 223,601 & 145,561 & 2 \\
\hline 16 & $\mathrm{M}+\mathrm{V}+\mathrm{P}+\mathrm{C} \& \mathrm{~B}$ & 2 & 130,209 & 9,743 & 139,953 & 335,930 & 195,977 & 1 \\
\hline 17 & $\mathrm{M}+\mathrm{C}+\mathrm{P}+\mathrm{C} \& \mathrm{~B}$ & 8 & 64,839 & 7,866 & 72,705 & 194,329 & 121,699 & 2 \\
\hline 18 & $\mathrm{M}+\mathrm{V}+\mathrm{C}+\mathrm{P}+\mathrm{Mul}+\mathrm{C} \& \mathrm{~B}+\mathrm{SR}$ & 1 & 157,317 & $21,805.38$ & 179,122 & 411,609 & 219,126 & 1 \\
\hline 19 & $\mathrm{M}+\mathrm{V}+\mathrm{C}+\mathrm{C} \& \mathrm{~B}+\mathrm{S} \& \mathrm{G}$ & 5 & 157,742 & 8,235 & 165,977 & 352,744 & 186,767 & 1 \\
\hline 20 & $\mathrm{M}+\mathrm{V}+\mathrm{C}+\mathrm{P}+\mathrm{C} \& \mathrm{~B}+\mathrm{S} \& \mathrm{G}$ & 3 & 139,602 & 12,166 & 151,768 & 330,986 & 179,288 & 1 \\
\hline 21 & $\mathrm{M}+\mathrm{V}+\mathrm{C}+\mathrm{C} \& \mathrm{~B}$ & 9 & 152,357 & 10,912 & 163,269 & 337,875 & 174,606 & 1 \\
\hline 22 & $\mathrm{M}+\mathrm{C}+\mathrm{P}+\mathrm{C} \& \mathrm{~B}+\mathrm{S} \& \mathrm{G}$ & 3 & 86,258 & 12,497 & 98,755 & 234251.7 & 135560 & 1 \\
\hline 23 & $\mathrm{M}+\mathrm{V}+\mathrm{F}+\mathrm{P}$ & 1 & 87278.26 & 14731.79 & 102,010 & 239,960 & 137,950 & 1 \\
\hline 24 & $\mathrm{M}+\mathrm{V}+\mathrm{C}$ & 7 & 81,344 & 12,657 & 94,000 & 205351.7 & 111351.2 & 1 \\
\hline 25 & $\mathrm{M}+\mathrm{C}$ & 2 & 38,441 & 17,990 & 56,431 & 140,807 & 84,376 & 1 \\
\hline 26 & $\mathrm{M}+\mathrm{V}+\mathrm{F}+\mathrm{C}+\mathrm{P}+\mathrm{C} \& \mathrm{~B}+\mathrm{S} \& \mathrm{G}$ & 1 & 122,755 & 8,115 & 130,871 & 277,495 & 146,624 & 1 \\
\hline 27 & $\mathrm{M}+\mathrm{C}+\mathrm{P}+\mathrm{Mul}+\mathrm{C} \& \mathrm{~B}+\mathrm{SR}+\mathrm{S} \& \mathrm{G}$ & 2 & 96,237 & 8,822 & 105,058 & 249,551 & 144492.6 & 1 \\
\hline 28 & $\mathrm{M}+\mathrm{V}+\mathrm{P}$ & 7 & 123,645 & 13,633 & 137,278 & 265,327 & 128,049 & 1 \\
\hline 29 & $\mathrm{M}+\mathrm{F}+\mathrm{C}+\mathrm{P}+\mathrm{C} \& \mathrm{~B}$ & 1 & 130,904 & 10,082 & 140,985 & 285,138 & 144,349 & 1 \\
\hline 30 & $\mathrm{M}+\mathrm{V}$ & 14 & 104,588 & 11,585 & 116,173 & 216,173 & 100,000 & 1 \\
\hline 31 & $\mathrm{M}+\mathrm{V}+\mathrm{C} \& \mathrm{~B}$ & 7 & 91,897 & 8,884 & 100,781 & 207,498 & 106,717 & 1 \\
\hline 32 & $\mathrm{M}+\mathrm{V}+\mathrm{F}+\mathrm{C}+\mathrm{C} \& \mathrm{~B}+\mathrm{S} \& \mathrm{G}$ & 1 & 208,163 & 16,494 & 224,656 & 353,033 & 128,377 & 1 \\
\hline 33 & $\mathrm{M}+\mathrm{V}+\mathrm{F}$ & 1 & 112167.63 & 8403.97 & 120,572 & 216,824 & 96,252 & 1 \\
\hline 34 & $\mathrm{M}+\mathrm{V}+\mathrm{Mul}+\mathrm{C} \& \mathrm{~B}+\mathrm{S} \& \mathrm{G}$ & 2 & 125,588 & 16,539 & 142,127 & 229,319 & 87,191 & 1 \\
\hline 35 & $\mathrm{M}+\mathrm{C}+\mathrm{P}+\mathrm{S} \& \mathrm{G}$ & 1 & 32,663 & 4,517 & 37,180 & 65,029 & 28,163 & 1 \\
\hline 36 & $\mathrm{M}+\mathrm{P}+\mathrm{C} \& \mathrm{~B}$ & 1 & 45,418 & 4,345 & 49,763 & 78,775 & 29,595 & 1 \\
\hline 37 & $\mathrm{M}+\mathrm{Mul}+\mathrm{C} \& \mathrm{~B}+\mathrm{SR}$ & 1 & 58,343 & 14,782 & 73,126 & 134,880 & 61,754 & 1 \\
\hline 38 & $\mathrm{M}+\mathrm{V}+\mathrm{P}+\mathrm{C} \& \mathrm{~B}+\mathrm{S} \& \mathrm{G}$ & 1 & 122,567 & 21,187 & 143,753 & 205,343 & 61,589 & 0 \\
\hline 39 & $\mathrm{M}+\mathrm{C}+\mathrm{P}$ & 3 & 16,517 & 13,771 & 30,288 & 35,710 & 5,484 & 0 \\
\hline
\end{tabular}

Source: Survey 2017

Journal of Experimental Biology and Agricultural Sciences

http://www.jebas.org 
Table 6 Farming pattern under rain-fed condition

\begin{tabular}{|c|c|c|c|c|c|c|c|c|}
\hline S No. & Category & Number of farmers & $\mathrm{VC}$ & FC & $\mathrm{TC}^{*}$ & GR* & $\mathrm{NR}^{*}$ & ROI \\
\hline 1 & $\mathrm{M}$ & 34 & 10160.72 & 312.14 & 10472.86 & 100665.46 & 90192.605 & 8.6120319 \\
\hline 2 & $\mathrm{M}+\mathrm{C}+\mathrm{P}$ & 27 & 20607.43 & 191.34 & 20798.77 & 115537.18 & 94896.651 & 4.5626087 \\
\hline 3 & $\mathrm{M}+\mathrm{PC}+\mathrm{C}+\mathrm{P}+\mathrm{C} \& \mathrm{~B}$ & 4 & 63477.77 & 1029.68 & 64507.49 & 240515.38 & 176170.65 & 2.7310108 \\
\hline 4 & $\mathrm{M}+\mathrm{C}+\mathrm{P}+\mathrm{S} \& \mathrm{G}$ & 9 & 24980.61 & 266.29 & 25246.90 & 125840.4 & 100622.1 & 3.9855227 \\
\hline 5 & $\mathrm{M}+\mathrm{C}+\mathrm{P}+\mathrm{C} \& \mathrm{~B}+\mathrm{S} \& \mathrm{G}$ & 23 & 65499.90 & 1238.64 & 66738.53 & 198949.18 & 132342.59 & 1.98 \\
\hline 6 & $\mathrm{M}+\mathrm{C}+\mathrm{P}+\mathrm{C} \& \mathrm{~B}$ & 33 & 59619.80 & 1388.52 & 61008.32 & 182605.2 & 121672.38 & 1.9943572 \\
\hline 7 & $\mathrm{M}+\mathrm{C} \& \mathrm{~B}$ & 11 & 49877 & 898.82 & 50775.81 & 170024.8 & 119249 & 2.35 \\
\hline 8 & $\mathrm{M}+\mathrm{P}+\mathrm{C} \& \mathrm{~B}$ & 11 & 64980.97 & 1431.22 & 66412.19 & 192640.1 & 126371.96 & 1.9028429 \\
\hline 9 & $\mathrm{M}+\mathrm{P}$ & 6 & 22134.6 & 205.22 & 22339.82 & 114395.3 & 92270.03 & 4.1302933 \\
\hline 10 & $\mathrm{M}+\mathrm{PC}+\mathrm{C}+\mathrm{C} \& \mathrm{~B}$ & 1 & 45891.74 & 700.5312 & 46592.28 & 174523.67 & 127931.39 & 2.745764 \\
\hline 11 & $\mathrm{M}+\mathrm{C}$ & 8 & 19795.29 & 183.96 & 19979.25 & 89847.26 & 69893.39 & 3.4982999 \\
\hline 12 & $\mathrm{M}+\mathrm{PC}+\mathrm{P}+\mathrm{C} \& \mathrm{~B}$ & 1 & 37380.35 & 804.46 & 38184.81 & 160858.75 & 122988.61 & 3.2208783 \\
\hline 13 & $\mathrm{M}+\mathrm{S} \& \mathrm{G}$ & 3 & 14775.71 & 159.79 & 14935.498 & 83707.361 & 68771.863 & 4.6045913 \\
\hline 14 & $\mathrm{M}+\mathrm{PC}+\mathrm{C}+\mathrm{C} \& \mathrm{~B}+\mathrm{S} \& \mathrm{G}$ & 1 & 70793.99 & 981.83 & 71775.82 & 236070.36 & 164294.55 & 2.2889959 \\
\hline 15 & $\mathrm{M}+\mathrm{C}+\mathrm{C} \& \mathrm{~B}+\mathrm{S} \& \mathrm{G}$ & 6 & 83789.57 & 1961.37 & 85750.93 & 193191.56 & 107440.63 & 1.2529384 \\
\hline 16 & $\mathrm{M}+\mathrm{C}+\mathrm{C} \& \mathrm{~B}$ & 2 & 70151.04 & 1159.65 & 71310.69 & 195936.08 & 124625.39 & 1.7476397 \\
\hline 17 & $\mathrm{M}+\mathrm{PC}$ & 2 & 12880.77 & 97.03 & 12977.69 & 57738.46 & 44760.77 & 3.449055 \\
\hline 18 & $\mathrm{M}+\mathrm{P}+\mathrm{S} \& \mathrm{G}$ & 1 & 18606.72 & 184.05 & 18790.772 & 92858.333 & 74382.228 & 3.9584445 \\
\hline 19 & $M+C \& B+S \& G$ & 4 & 51444.77 & 907.46 & 52352.23 & 146047.71 & 93695.49 & 1.7897134 \\
\hline 20 & $\mathrm{M}+\mathrm{PC}+\mathrm{C}+\mathrm{P}$ & 2 & 17893.35 & 153.63 & 18046.982 & 55786.112 & 37739.129 & 2.0911601 \\
\hline 21 & $\mathrm{M}+\mathrm{PC}+\mathrm{P}$ & 1 & 20038.54 & 165.31 & 20203.854 & 66340 & 46136.146 & 2.2835319 \\
\hline 22 & $\mathrm{M}+\mathrm{P}+\mathrm{C} \& \mathrm{~B}+\mathrm{S} \& \mathrm{G}$ & 1 & 105,055 & 1,786 & 106840.55 & 206491 & 99650.449 & 0.9327025 \\
\hline
\end{tabular}

Source: 2017

In the below table 5 and table6; $\mathrm{M}=$ Mango; $\mathrm{OF}=$ Other Fruits; $\mathrm{F}=$ Flowers; $\mathrm{PC}=$ Plantation-crops; V=Vegetables; $\mathrm{C}=\mathrm{Cereals}$; $\mathrm{P}=\mathrm{Pulses}$; Mul= Mulberry; C \& B= Cow \& Buffalo; SR= Silkworm Raring; S \& G= Sheep \& Goat

*We have used the below-mentioned formulae for calculating Total cost (TC), Gross Returns (GR), and Net Returns (NR) per category to get the per acre/ per herds/ per $100 \mathrm{DFL}$

Average TC or GR or NR of each category = Summation of TC or GR or NR of all the respondents in the category divided by the summation of the total landholdings of the respondents in the category

Average TC or GR or NR of each category = Summation of TC or GR or NR of all the respondents in the category divided by the summation of the Herds in the category

Average TC or GR or NR of each category = Summation of TC or GR or NR of all the respondents in the category divided by the summation of the DLF in the category which is further multiplied by 100 to get the average per $100 \mathrm{DFL}$.

\section{Conclusion}

Both economies of scale and scope for smallholding mango growers in Srinivaspurtaluk of Kolar district in Karnataka is categorized as dry land. Mango cultivation gives the highest returns on investment for both kinds of respondents in rain-fed and irrigated conditions. However, growing cereals and pulses give food security. The most staple cereal crop is ragi which could be easily grown in kharif with mere or less irrigation. It does not require too much water like paddy. Raring livestock is necessary for residual soil fertility management.

The respondents grow several crops to meet the green fodder requirements for the HF cows and Murrah buffaloes which also consume some water for irrigation as few crops like jowar, corn, and hybrid maize. Therefore, raring the HF cows and Murrah buffaloes could be more feasible in irrigated conditions. Respondents under rain-fed conditions could also tame more domestic cows and try zero-budget natural farming (ZBNF). ZBNF could help those farmers to go organic and have a reasonable yield. Organic food has more value in the market which will enhance the profit of the rain-fed farmers. It will also help them for betterintegrated farming as the domestic cows do not have green fodder requirements and care as the HF cows. Vegetables do have high returns but they are highly price-elastic and variable cost is also high. So, growing the only vegetable without plantation crops, other fruits, cereals, and pulses cannot give sustainability to the mango growers. Therefore, it could be grown for profitability in one of the seasons. Marigold could be grown during festival seasons by irrigated farmers for better returns. Moreover, raring sheep and goats and domestic cows are feasible for the rain-fed farmers as the fodder generated from the cereals and pulses grown during kharif season could be used. Fruits like papaya and plantation crops like coconut gives high net returns. It also does not consume more space. If near to fencing of the farmland farmers grow coconut and papaya could enhance their income. 


\section{Conflict of Interest and Financial Disclosure}

There is no conflict of interest among the authors and authors did not receive and financial support from anywhere to conclude the research.

\section{References}

APEDA (2020) Available at http://apeda.gov.in/apedawebsite/ six_head_product/FFV.htm access on $25^{\text {th }}$ February 2021

Beattie A (2020) Available at https://www.investopedia.com/ articles/basics/10/guide-to-calculating-roi.asp\#: :text=ROI\%20is\% 20calculated $\% 20$ by $\% 20$ subtracting,finally $\% 2 \mathrm{C} \% 20$ multiplying $\% 2$ 0it\%20by\%20100 access on $5^{\text {th }}$ September, 2020.

Behera UK (2012) Multiple Water Use Protocols in Integrated Farming System for Enhancing Productivity. Water Resources Management 26(1): 2605-2623.

Chen J (2020) Available on https://www.investopedia.com/terms/r/ returnoninvestment.asp. (J. Mansa, Ed.) Access on $23^{\text {rd }}$ October, 2020.

Devi D (2016) Influence of Nitrogen-Fixing and Phosphorus Solubilizing Bacteria on the Performance of Kulthi (Dolichos Biflorus). Unpublished thesis submitted to the Indira Gandhi Krishi Vishwavidyalaya, Department Of Agricultural Microbiology. Raipur.

Gill MS (2009) Integrated farming system and agriculture sustainability. Indian Journal of Agronomy 54(2): 128-139.

Indian Horticulture Database (2011) Available at http://nhb.gov.in/ access on $25^{\text {th }}$ February 2021.

Khadse A, Peter MR (2019). Zero Budget Natural Farming in India - from inception to institutionalization. Agroecology and Sustainable Food System 43(7-8): 848-871.

Mathew M, Rao YS (2012) Tamarind (Tamarindus indica L.) research - a review. In: Mathew M (Ed.), Handbook of Herbs and Spices, Sawston, United Kingdom: Woodhead Publishing Limited 1: $513-531$
Mehjabeen, Saravanadurai, A. (2020). Trends in Profitability Amidst Production Constraints Encountered by Smallholder Mango Growers - a case of India. Asian Journal of Agriculture and Rural Development 10(1): 336- 345.

Motkuri V (2020) Vegetable Consumption in India: Supply Chain and Prices. Available at www.mpra.ub.uni-muenchen.de: https://mpra.ub.uni-muenchen.de/101979/1/MPRA_paper_101979 .pdf access on January 28, 2020.

National Mango Database (2020) Available at https://mangifera.res.in acces on $29^{\text {th }}$ April 2020.

Radhamani SB (2003) Sustainable integrated farming systems for drylands - A review. Agricultural Reviews 24(3): 204-210.

Shetty M (2019). Farmers cultivate Nilgiri trees despite a ban. Avialable at www.newsnet.iijnm.org: http://newsnet.iijnm.org/ farmers-cultivate-nilgiri-trees-despite-a-ban/ access on October 21, 2020.

Shivakumar HK, Chandra KVT (2016) District Irrigation PlanKolar District. Bangalore: Government of Karnataka.

Singh J, Gangwar B, Pandey D, Kochewad S (2011) Integrated Farming System Model for Small Farm Holders of Western Plain Zone of Uttar Pradesh. Indian Council of Agricultural Research, Project Directorate for Farming Systems Research. Meerut: Project Director, Project Directorate for Farming Systems Research, Modipuram, Meerut, India.

Smathers M (2017) Available at https://sciencing.com/calculateelectrical-consumption-motor-7464755.html access on October 22, 2020.

The Director of Statistics (2015) Horticulture Crop Statistics of Karnataka State at a Glance. Bangalore: The Directorate of Horticulture.

Zarzycki N (2020) How To Calculate Straight Line Depreciation (Formula). Available at https://bench.co/blog/accounting/straightline-depreciation/ access on October 23, 2020. 